\title{
CLASSEMENTS, CAPITALISME ACADÉMIQUE ET AFFECTS DES CHERCHEURS EN GESTION
}

\author{
Hugo Letiche, Geoff Lightfoot et Simon Lilley \\ University of Leicester (UK)
}

\section{RÉSUMÉ}

Cet article traite de performativité. Au premier degré, de la performativité des notations des articles et des classements des universités dans le cas du Royaume-Uni, de la littérature de protestation et d'accusation qu'ils ont engendrée. Au second degré, seront examinées les conditions sociales ou politiques qui ont produit ces classements et notations, notamment le " capitalisme académique » et la prolétarisation pensés par Bernard Stiegler. Mais, en fin de compte, au troisième degré, nous estimons que les classements et les notations ont surtout un impact sur les affects des chercheurs. Classements et notations sont des textes performatifs : ils créent une mentalité, un sentiment d'identité, voire un environnement de vie, que nous réfléchirons depuis l'intérieur. A partir de textes réflexifs et auto-ethnographiques, nous nous demandons ce que le chercheur académique devient, quel est le ton subjectif de son état d'esprit dans le régime des notations et des classements, ce qu'engendre cette performativité dans l'économie de la connaissance ?

\section{MOTS CLÉS}

Évaluation de la recherche, prolétarisation, capitalisme académique, tournant vers les affects, performativité, Stiegler.

\section{INTRODUCTION}

Une abondante littérature sur l'évaluation des recherches et des revues a mis en évidence leurs effets destructeurs sur la recherche et sur les chercheurs. Mais cette littérature attribue trop rapidement l'omniprésence des classements à la politique néolibérale et à la tendance de vouloir tout diriger, y compris les universités, comme des entreprises. Le paradoxe est que le carriérisme et le cynisme extrêmes ainsi dépeints ne correspondent pas à la description de l'entreprise créative de l'économie de la connaissance (Hughes, 2011 ; Marcus, 1995). Et l'ironie est que certains des plaintifs ont très bien réussi dans les notations et les classements justement en publiant leurs plaintes véhémentes. Si le capitalisme académique est réellement produit par les exigences de l'économie de la connaissance (Sum \& Jessop, 2013), il en est un sous-produit 
inquiétant notamment du fait des affects ainsi engendrés. Si nous voulons réussir continuer à prospérer dans l'économie de la connaissance à venir, nous devrions réfléchir aux affects que l'organisation académique engendre chez les chercheurs.

Du fait de tous les arguments avancés contre lui, on ne peut que s'étonner de la prédominance des classements et des notations. Le capitalisme académique et l'approche de Bernard Stiegler en donne une explication possible. Notre thèse est que la question clé est celle des affects provoqués par la culture des notations et classements. Et que nous devons nous en préoccuper pour maintenir notre place dans l'économie de la connaissance.

Notre argument comprend trois étapes. Premièrement, il synthétise les critiques anti-classement publiées dans les revues britanniques. Ensuite il pointe les forces du « capitalisme académique »dominantes dans le monde universitaire et examine comment la «prolétarisation » et l' « hyperdiachronisation » éclairent ces évolutions. Enfin, il revient sur la performativité affective des évaluations des revues et des universités, tentant d'en dégager certaines significations pour la recherche en gestion. Notre méthode repose sur l'analyse textuelle selon trois degrés de sens de Roland Barthes (texte/intention/signification), recourant pour le troisième à de l'autoethnographie.

\section{SYSTÈME(S) DE CLASSEMENT BRITANNIQUE(S)}

II existe une vaste littérature sur les systèmes de classement, notamment sur le guide de l'ABS (Association of Business Schools rating of journals) et le RAE/ REF (Research Assessment Exercise devenu Research Assessment Framework) (RAE, 2006, 2008 ; Cooper \& Otley, 1998). Cette littérature est essentiellement britannique. II semble que nulle part ailleurs de tels systèmes aient eu autant de poids et de légitimité qu'au Royaume-Uni (Carter, 2008). Tous les observateurs semblent convenir que la montée conjuguée de ces deux processus a radicalement changé le milieu universitaire (Strathern, 1997).

Précisons que les deux systèmes sont distincts. Le guide de l'ABS n'a pas de statut ministériel ou officiel. Les membres du groupe consultatif de la RAE/REF, 
qui jugent la qualité des travaux, insistent sur le fait qu'ils ne suivent pas le guide de l'ABS dans leurs délibérations. Le guide classe les revues en $4^{*}, 3^{*}$, $2^{*}$ et $1^{*}$. II est présupposé que les articles dans une revue $4^{*}$ sont tous de qualité $4^{*}$ et c'est la raison pour laquelle elle est une revue $4^{*}$. La logique est circulaire: une revue $4^{*}$ est une revue où des articles $4^{*}$ sont publiés (Macdonald \& Kam, 2007).

Grosso modo, $4^{*}$ se traduit par « renommée mondiale », 3* par « excellence », $2^{*}$ par « médiocre » et $1^{*}$ par « insignifiant » ${ }^{1}$. Une enseignante-chercheur est au maximum du succès si elle publie quatre articles $4^{*}$ tous les six ans. On ne présente que quatre articles à la REA/REF, aussi nous entendons une collègue s'écrier : " J'ai mes quatre ! » : elle n'a plus rien à faire puisque plus rien d'autre ne sera pris en compte dans l'évaluation (Lilley, 2017). La recherche devient un effort pour réaliser quatre articles $4^{*}$ et non plus une activité de débat, de découverte ou une affaire de lecture.

Le RAE/REF est un processus ministériel de classement des départements. Encore une fois, l'échelle est à quatre niveaux, et ce sont les résultats en recherche des départements (par exemple de gestion) qui sont évalués ; aucune note individuelle n'est révélée. Les départements classés 4 * obtiennent plus de financement pour la recherche. Dans le dernier exercice (REF 2014), $1^{*}$ et $2^{*}$ ne donne pas lieu à financement gouvernemental pour la recherche, alors que les $4^{*}$ ont obtenu quatre fois plus de financement que les trois étoiles. Chaque département présente jusqu'à quatre publications par membre du personnel. II s'agit presque toujours d'articles. Un groupe de pairs évalue le travail soumis sur l'échelle à quatre niveaux. Les membres de la commission n'ont pas la possibilité de lire toutes les soumissions - il y en a juste trop et pas assez de temps (Anonyme, 2015). Ce mode de valorisation a généré bien des controverses. Les membres de la commission ont insisté sur le fait qu'ils regardaient le travail et non pas seulement dans quel journal il est paru. Par exemple, dans un cas la commission en gestion a fait l'éloge de Ephemera (qui à l'époque était $1^{*}$ ) et compté en moyenne les articles de JMS (un journal $4^{*}$ ) comme $3^{*}$ (Tourish \& Willmott, 2014).

\footnotetext{
1 Les définitions officielles sont : $4^{*}$ " leader mondial "; $3^{*}$ " excellent à l'échelle internationale »; $2^{*}$ « reconnu internationalement »; $1^{*}$ « reconnu à l'échelle nationale ».
} 
La littérature critiquant le Guide de l'ABS et le RAE/REF dénonce principalement les effets induits sur les pratiques de la recherche et l'identité des enseignants chercheurs dans les universités britanniques, tout particulièrement dans les départements de commerce. Faire de la recherche, se faire publier, la «bonne recherche » auraient été changés. Les « habits neufs du chercheur », tels que décrit récemment dans un article d'Organization $\left(4^{*}\right)$, l'ont laissé nu, exposé, plein d'anxiété et souvent assez autodestructeur. L'auteure, Karen Lee Ashcraft (2017), a exploré l'affect-classement, autrement dit ce que le régime de classification fait à la subjectivité des chercheurs. Elle livre le panorama suivant:

1. Se fonder sur les classements de revues minimise d'autres formes d'évaluation universitaires en faveur d'une liste universelle, qui devient un indicateur universel de qualité de par la répétition de son utilisation (remplaçant une évaluation complexe de la qualité).

2. Le prestige d'une revue devient plus important que son contenu et tend vers son autoentretien indépendamment du contenu (par exemple le taux de rejet augmente avec l'accroissement du nombre de soumissions).

3. L'injonction à tous de viser les meilleures revues finit par faire exploser la charge de travail du journal à mesure que les soumissions proviennent de plus bas dans la hiérarchie.

4. Cette charge de travail s'intensifie continuellement puisque : (a) les universités mettent la barre plus haut pour la productivité en recherche et (b) les revues réduisent les temps de décision - chacune pour améliorer leur performance respective dans «le classement ».

5. La demande de soumettre dans les meilleures revues produit une aversion pour le risque intellectuel et, en fin de compte, une homogénéisation, écrêtant les marges radicales. Même les articles des revues secondaires ou alternatives gardent le biais conservateur de l'émulation des revues prestigieuses.

6. Les revues de haut-niveau privilégient les chercheurs anglophones et favorisent les traditions inspirées par les sciences sociales nord-américaines.

7. La recherche se réduit au jeu des publications. Nous nous préoccupons davantage des jeux politiques internes que de la participation aux idées et leurs implications générales.

8. Le jeu de la publication détruit la collégialité et engendre la démoralisation dans une économie des « étoiles académiques » (Solomon, 2008). « Pas étonnant que l'une des émotions prédominantes dans l'université néo-libérale soit le ressentiment... » (Meyerhoff et al., $2011:$ 492).

9. Le jeu de la publication fournit de nouveaux plaisirs personnels (par exemple passer 
l'évaluation, l'accroissement de sa valeur de marché). Les plaisirs antérieurs, comme son accomplissement dans des projets qui ont du sens, ne se maintiennent que par l'attachement, destructeur, à jouer selon La Règle.

Encadré 1 : Effets de subjectivité liés aux affects suscités par les classements (Ashcraft, 2017, p. 40)

Quelques commentaires :

1. Les revues $4^{*}$ adoptent une approche en très grande majorité positiviste et quantitative. Les essais, la réflexion théorique et l'expérimentation formelle sont devenus presque impubliables (Hall, 2011 ; Merilainen et al., 2008). Ironiquement, l'impact de la recherche pâtit du fait que les revues académiques en deviennent incompréhensibles pour la plupart des professionnels ou des étudiants. Et pourtant "l'impact », au sens du REF, désigne spécifiquement un lien démontrable entre une publication préalable et un changement de pratique ou de mode d'interprétation dans le monde non académique.

2. Écrire dans une revue demande de beaucoup citer la revue cible (cela élève leur indice de citation) et de s'assurer que vous vous conformez à sa ligne éditoriale. Le chercheur écrit pour être accepté et gagner des bons points professionnels - et non pour lancer un débat, défendre un autre point de vue ou prendre position.

3. L'examen par les pairs est devenu de plus en plus problématique, car nous avons très peu de temps pour le faire et ce travail ne compte pas dans les évaluations. L'examinateur sera de moins en moins enclin à accepter une perspective inhabituelle ou concurrente - il sera tenté (consciemment ou non) de défendre sa position et son espace de publication.

4. Tout le monde commence par soumettre ses articles aux mêmes revues $4 *$. Le choix de la revue n'est pas déterminé par « la pertinence » mais par le prestige. Si votre recherche se situe dans une sous-discipline, ou plutôt récente, « votre » revue sera mal classée. Combien d'articles sont soumis à des revues que leurs auteurs n'auraient jamais (spontanément) choisi de lire ! 
5. L'intensification du travail est le résultat de l'action conjuguée des universités, des revues et des universitaires chacun en compétition pour les meilleures évaluations. Tous les dirigeants d'université et d'école veulent que l'évaluation de "leur » établissement progresse. Ainsi, ils exigent davantage de leurs membres et ferment les départements qu'ils estiment plus faibles. La recherche est guidée par les institutions et la recherche de performance, avec de plus en plus d'exigences pour le corps professoral (Fournier et Grey, 2000).

6. Les meilleures revues en gestion ont un fort biais étatsunien. Vous devez citer le travail américain, reproduire leurs méthodes et accepter (implicitement) les normes économiques et sociales de cette société.

7. Faire de la recherche veut désormais dire se faire publier. Ce n'est plus l'importance de la question de recherche mais le résultat qui compte. Cela favorise les stratégies de recherche à court terme, faciles à mettre en œuvre. Sécuriser son emploi et sauvegarder son temps de recherche sont devenus cruciaux (Cederstrom \& Hoedemaekers, 2012).

8. Les meilleurs chercheurs se lisent et commentent leurs papiers entre eux bien avant qu'ils ne soient soumis. Les "vedettes " affinent leurs pratiques au sein d'un réseau coopératif d'entraide. Mais ceux qui ne font pas parti des meilleurs en sont exclus. La recherche s'apparente à l'atelier de l'artiste au 17ème siècle. Le maître fournit quelques touchesclé (par exemple, quant à l'expressivité) et les subordonnés font tout le travail de routine

9. Le succès est pour le succès, et règne la marchandisation de soi. Votre valeur c'est celle de votre dernier article accepté. La recherche devient un concours pour le statut et la profession perd son sens de la communauté. L'évaluation par les pairs est un jeu concurrentiel. Le contenu est le plus grand perdant.

II reste que certains effets (potentiellement) positifs peuvent être associés au système de classement : 
1. Si vous savez écrire, vous pouvez l'emporter. II y a probablement plus de pensées et d'idées critiques publiées dans le système actuel qu'il n'y en aurait sinon (Butler \& Spoelstra, 2014). Un argument bien formulé, appuyé par une connaissance approfondie de la littérature et par une observation empirique (même qualitative), le rendra (éventuellement) imprimé. Sous le régime antérieur, il y avait moins de transparence, moins de publications et plus de favoritisme qu'aujourd'hui.

2. Devoir rendre des comptes de sa recherche, même dans un système imparfait, est justifié. Les chercheurs sont payés pour mener des recherches sur des fonds parapublics. Bien que le pourcentage du financement provenant du gouvernement soit de moins en moins élevé, une responsabilisation est indispensable. La "pertinence" ou la valeur sociale des travaux universitaires doit être discutée et examinée (Furlong, 2011).

Le paradoxe apparent est que les incitations financières (gouvernementales) sont insignifiantes. Dans notre université anglaise, moins de $5 \%$ du budget est lié au RAE. À l'Université de Warwick, considérée comme très efficace pour attirer des fonds liés à RAE, le chiffre tourne aussi autour de $5 \%$. Ainsi les classements ne constituent pas vraiment un facteur financier crucial. À £9 000 par an et par étudiant de première année, on peut augmenter les frais de scolarité et compenser tout déficit du budget de recherche. De plus, les revenus de la recherche ne couvrent qu'environ $85 \%$ des coûts réels. En termes nets, au Royaume-Uni, la recherche universitaire est presque toujours un centre de coûts et, au mieux, une source d'exposition financière permanente qui doit être couverte par d'autres sources.

En résumé, si les revues $4^{*}$ récompensent une certaine rationalité technique, elles ne valorisent pas nécessairement le "sérieux ». Des questionnements comme ce qu'est l'équité, comment réaliser une société juste ou ce que demande l'épanouissement des hommes sont enterrés dans la ruée vers le succès. Les classements et les évaluations vénèrent « la déesse putaine de la réussite » (James, 1920). L'histoire racontée dans les revues $4^{*}$ n'est (presque) jamais un récit de défaite, de tragédie ou de perte. Pour vendre la performance managériale les textes ressemblent trop souvent à ceux de consultants 
essayant de vendre des solutions réussies. À notre avis pourtant les chercheurs universitaires devraient explorer les comportements et les relations plus en profondeur, décrire tant les réussites que les échecs, examiner le sens et les desseins, révéler les tendances et les hypothèses, montrer les biais et déstabiliser les idées préconçues.

Dans l'ensemble, l'évaluation de la performance des écoles de commerce britanniques, avec son obsession des $4^{*}$, est plutôt négative (Naidoo, 2016) ${ }^{2}$. Le classement de la recherche crée une recherche pour classer, qui ne sert pas la créativité, le renouvellement intellectuel ou l'intégrité académique (Tadajewski, 2016). Les systèmes de notation ne produisent que des platitudes très coûteuses: il fait peu de doutes que Cambridge et Oxford soient des centres d'excellence, les évaluations coûtent des centaines de millions de livres pour « découvrir » de tels faits (Sayer, 2015).

Hugh Willmott, en tant que membre senior de la RAE et professeur de recherche $4^{*}$, a écrit à de nombreuses reprises que nous devrions tout simplement ignorer le système de classement des revues (cf. 2014). Mais ce n'est pas ce qui se passe. Pourquoi ? Quels sont les besoins fondamentaux servis pas les systèmes de notation ? Certes, les classements facilitent le travail des directeurs d'université, car ils peuvent embaucher, promouvoir et licencier sans avoir à prendre une responsabilité intellectuelle pour leurs actions ou au nom des institutions dont ils sont censés être responsables. Mais nous ne pensons pas que ce soit la principale raison d'être du système.

Par conséquent, de quoi s'agit-il vraiment, si ce n'est pas en fait d'excellence en recherche ou de financement (supplémentaire) ? Nous allons le voir, la réponse semble être que le «budget de recherche » est un «budget marketing »! Tout tourne autour du statut et de (sa propre) importance. Les classements des articles et des établissements déterminent dans quelle mesure une personne ou une université est « attractive ».

\footnotetext{
${ }^{2}$ Un commentaire moins critique du classement se trouve dans Rowlinson et al. (2011) et Morris et al. (2009).
} 


\section{CAPITALISME ACADÉMIQUE,}

HYPERDIACHRONISATION

Le « capitalisme académique » est un concept lancé principalement par Sheila Slaughter et ses co-auteurs (Slaughter \& Leslie, 1997 ; Slaughter \& Rhoades, 2000 \& 2004 ; Cantwell et al., 2014). II place l'université contemporaine dans la logique de l'économie du savoir et de son idéologie néolibérale (Ward, 2012). Au moins en partie, il s'inspire de la présentation du néolibéralisme de David Harvey (2005) qui elle-même doit beaucoup à Duménil et Lévy (1998, 2000). Ces derniers expliquent que le capitalisme moderne a connu quatre crises : celle des années 1890, de 1929, des années 1970 et de 2008. Celle des années 1970 a été "résolue " par une première vague de politiques néolibérales, souvent identifiée avec Thatcher et Reagan. Celles-ci ont fait reculer les garanties liées à la santé, au logement, à l'éducation et aux retraites. Et après 2008, ce recul s'est intensifié, tout comme la croissance de la richesse des plus riches. Duménil, Lévy et Harvey insistent sur le fait que les évolutions politico-économiques contemporaines post-1970 reposaient sur une base idéologique. Après tout, Thatcher et Reagan ont été (répétitivement) élus.

Après la Seconde Guerre mondiale, la condition sine qua non de l'université était le "bien-être général » et I' "intérêt commun ». La connaissance s'identifiait avec les Lumières, la conscience commune et le progrès humain. L'idéal académique était collégial et contributif ; si « les communs » existaient quelque part, c'était sur les campus. Les idéologies de l'époque, de la socialdémocratie à l'existentialisme, célébraient (au moins la possibilité de) la responsabilité et de l'existence authentique. La croyance que «la connaissance rend libre " a justifié, d'une part, un mode de vie très individualiste et, d'autre part, la conviction que l'effort de chacun avait une valeur sociale.

Pour les universitaires, les années 1970 ont précipité une période nouvelle et bien moins favorable du « capitalisme académique ». Sur le plan idéologique, I' « économie de la connaissance » claironnait l'importance de l'université, une importance telle qu'elle devait être gérée, contrôlée et disciplinée. Le néolibéralisme cherchait à réformer tous les services (publics), à les rendre « efficaces » et orientés vers le marché. La crise économique des années 1970 
impliquait beaucoup moins d'argent public pour l'éducation. Et pour sortir de l'ornière de la stagflation, il fallait innover davantage.

En fait, les universitaires ont été chargés d'en faire plus pour moins. La recherche devait être « pertinente », c'est-à-dire utile à l'industrie. Une nouvelle classe de gestionnaires a été engagée pour s'assurer que cela se produirait. L'enseignement devait coûter moins cher - ce qui se traduisait souvent par des cours dispensés par des étudiants ou des vacataires. Ceux qui ont eu la chance de trouver un poste permanent devaient publier beaucoup plus qu'avant et mieux servir les intérêts économiques. Pour une petite élite, la nouvelle situation a conduit à des salaires très élevés et des privilèges jusqu'alors inconnus. Mais pour la plupart, il s'agissait d'une pure intensification du travail : il s'est agi de faire plus pour moins de salaire, de prestige ou de sécurité d'emploi. Certains ont protesté contre la « déqualification » des universitaires et l'intensification de la charge de travail (Gonzales et al., 2014). Ils déploraient les coûts humains, familiaux, de santé (mentale) et de qualité de vie (Boesenberg, 2015). Mais pour ceux qui arrivaient là, la réaction dominante a été de « faire avec ».

Où le « capitalisme académique » aboutit-il ? Si nous suivons la pensée de David Graeber (2011), la réponse doit être à la financiarisation de l'université. Ben Jessop (2017) en a esquissé l'imaginaire. Le «cloisonnement des communs académiques ॥ (Jessop: 2017, p.860) rend rétrograde la recherche au service du bien public. La croyance néolibérale en « l'individu intéressé par lui-même », qui agit comme un optimiseur rationnel poursuivant ses propres besoins et intérêts, a triomphé. Le monde académique a perdu ses anciennes ressources idéologiques en tant qu'acteur du bien-être général. Le néolibéralisme, qui se concentre sur le succès dans les affaires, célébrant la concurrence et l'esprit d'entreprise, a pris le dessus. II considère justifiées les énormes différences entre ceux qui réussissent et ceux qui échouent. Le consensus et la coopération sont considérés comme improductifs, inefficaces et répressifs. Le milieu universitaire a dû apprendre de nouvelles valeurs et à s'adapter à une culture bien différente. Et c'est exactement ce qu'il a fait, et ceci très rapidement. Le capitalisme académique est devenu la norme : avec des spin-offs dirigées par des professeurs, des universitaires qui font du consulting, 
des brevets et droits d'auteur détenus par les universités et des dotations d'entreprises déterminant les agendas de recherche. Les professeurs doivent vendre leurs propositions de recherche pour pouvoir les financer. Les universités projettent des images de réussite afin de renforcer leurs liens avec les entreprises. Le statut d'élite joue un rôle clé dans l'obtention du financement, dans l'attraction des meilleurs et des plus brillants ainsi que pour l'avancement professionnel. Tout et tout le monde ont recours à de l'autopromotion pour réussir.

Pour comprendre cette évolution, on peut se référer à la sociologie du contemporain de Bernard Stiegler, qui étudie le nivellement par le bas de la société. Pour lui, les changements des années 1970 ont accéléré le rôle croissant du marketing. Alors que le fordisme était centré sur la croissance économique et la production, le post-fordisme était (hyper)consummériste. Les symboles extérieurs du statut et de la réussite, ou du style de vie, sont devenus prioritaires.

Dans la logique consumériste, les articles $4^{*}$ existent pour promouvoir la place d'élite des professeurs seniors et des départements. Les publications n'ont pas à être lues, étudiées ou débattues. L'objectif est de réussir dans les évaluations, et non de communiquer des idées d'un chercheur à l'autre ou d'une génération à l'autre. Le but de communication - surtout de génération en génération - a été supprimé par l'objectif de marketing (Stiegler, 2010a). Être « numéro un » ou « excellent » est universellement communicable, et universellement sans contenu (Butler \& Spoelstra, 2014). La recherche est devenue une forme de publicité institutionnelle en défense de la hiérarchie existante. Le système de classement est le contexte institutionnel dans lequel tout cela a lieu. Sans le système des classements, les universités pourraient servir de lieux pour des idées, des débats et des recherches, mais elles ne sauraient aussi puissamment produire des marques. Ainsi, notre argument, inspiré par Stiegler (2013, 2016), est que le branding : (1) détruit le processus (intergénérationnel) de la transmission des idées et/ou de la socialisation d'une cohorte à l'autre; (2) fournit une preuve d'exclusivité - la recherche n'a pas besoin d'être lue ou mise en œuvre mais vise à exclure le plus grand nombre de son statut d'élite 
Les cycles de vie de la connaissance sont courts, le " canon » théorique et méthodologique est instable. Les vieilles connaissances sont démodées. Le but est d'être « à jour », « contemporain » et « actuel » - en d'autres termes «à la mode » ou «à la minute». L'université n'est plus dépositaire de connaissances éprouvées. La nouveauté et non la continuité est son habitus. Mais comme l'a soutenu Stiegler, le résultat est l'aliénation de l'un par l'autre (2010a). La culture humaniste des Lumières, développée au cours des trois cents dernières années, est rejetée car non contemporaine. Marx-WeberDurkheim-Freud ou Kant-Hegel-Husserl-Heidegger sont juste démodés et supposés non pertinents. La culture, les traditions et les valeurs européennes ne sont plus le fondement de notre connaissance. L'attention se porte sur les idées à la mode, comme le commerce électronique et les technologies de l'information, ou encore le leadership et l'entreprenariat, et oublie plus ou moins la théorie des organisations, l'histoire de la pensée gestionnaire ou l'éthique. Publier sur ces domaines et les big five (Google, Microsoft, Facebook, Amazon, Apple) est « hot », alors que la réflexion théorique, les études sur le travail ou l'économie politique ne sont pas ce que désire le marché. Chacun finit verrouillé dans sa mode, étranger des autres. II y a une insuffisance de continuité ; les connaissances passées ne sont pas transmises de manière adéquate. Les erreurs sont trop facilement refaites, les connaissances acquises inutilement perdues. Et la camaraderie est presque impossible.

Selon Stiegler, la société (hyper)consommatrice guidée par le marketing a après 2008 été remplacée par des exigences de performativité toujours plus radicales. Dans les années 1970, l'ordre social du tout commerce a été destructeur, mais aujourd'hui c'est le sens humain de l'action qui est en grande partie détruit. Après 2008, ce serait l'humain lui-même qui a changé. Les forces du marketing et de l'hyperconsommation ont réussi à redéfinir la nature même de la conscience et de l'identité.

Dans ses travaux antérieurs, Stiegler (1998, 2008, 2010b) était proche de la croyance simondonnienne de la possibilité d'utiliser la technologie (principalement les machines) pour le bien de l'homme. La pensée de Stiegler a conservé ses racines dans la conception de l'individuation de Simondon (Letiche \& Moriceau, 2017; Scott, 2014), selon laquelle ce que nous devenons 
en tant qu'identité, personne, conscience et capacité d'agir prend forme dans le processus d'individuation que nous traversons. Mais l'individuation a changé. Selon Simondon, l'individuation a lieu dans le pré-individuel : immergée dans ce que le social, technologique, politique, économique et biologique contiennent en puissance. L'individu ne maîtrise pas son individuation, le préindividuel fournit les opportunités et les buts d'individuation. L'individuation est un processus complexe, relationnel et encastré. Les changements historiques ou d'époque dans le pré-individuel sont de ce fait des événements cruciaux.

Stiegler soutient que la prolétarisation représente justement un changement fondamental dans le pré-individuel, c'est-à-dire que ce que les personnes sont, et peuvent être, a changé. Le terme "prolétarisation » est bien sûr une référence à Marx. Au XIXe siècle, les artisans perdirent leur savoir-faire suite à la mécanisation de leur travail et une main d'œuvre non qualifiée, avec l'utilisation de nouvelles machines, a pris le relais. Les métiers disparurent, les compétences et connaissances du passé devinrent sans importance. Les habilités et savoirs autrefois prisés perdirent toute leur valeur. Les ouvriers de l'usine, qui remplaçaient les artisans, ont été laissés avec un travail routinier stupide.

Dans la tradition de Simondon (et de Hannah Arendt, 1998), la distinction entre l'œuvre et le travail est cruciale pour la formation de l'identité. L'œuvre doit être entendue au sens des «œuvres d'art»; elle implique un projet ou un art personnel, ou encore une mission. L'œuvre est une source essentielle de l'individuation, tandis que le travail salarié en grande partie bloque la créativité, la formation de l'identité et le sentiment d'estime de soi. Les universitaires ont généralement compris leur travail comme la production d'œuvres. Ce contre quoi les critiques du capitalisme académique protestent, c'est la prolétarisation. L'université connaît le même chemin que les ateliers d'artisanat du passé.

Selon Stiegler, la prolétarisation en tant que processus de déqualification s'est étendue à toute la société : pensée critique, conscience réflexive, identité personnelle et conscience de soi sont sacrifiées à la consommation de masse. Ce que l'on voit, ce à quoi on s'identifie et aspire, ce qu'on valorise et désire, tout est normalisé. Le désir a été aplati et uniformisé, noyé dans des pulsions non individuées, entrainant la perte de la capacité socio-psychologique d'agir. 
L'individuation est prolétarisée, c'est-à-dire les possibilités mêmes de l'identité, de la conscience et du bien-être. Le soi est déqualifié - il perd sa capacité de jouir, de créer, de se sentir vivant et de partager. Dans "l'hypersynchronisation », les identités sont produites à l'échelle industrielle, avec perte de la possibilité même de l'individuation (Dillet, 2017).

En réaction, ce que Stiegler appelle l' « hyperdiachronisation » ou le repli sur son sous-groupe, répond à la désintégration de la possibilité même d'une identité partagée. Pour s'opposer à une attaque de sa formation identitaire, la personne peut se refermer sur de petits groupes hautement auto-protecteurs, qui mettent en valeur leur singularité. Ces groupes réagissent avec hostilité au " monde extérieur » et se considèrent comme victimes sous la menace du capitalisme. De telles attitudes sont courantes dans la littérature sur le capitalisme académique, en particulier lorsqu'elle dépeint l'universitaire (junior) comme victime, à l'image de la revue Workplace: A Journal for Academic Labor (Boesenberg, 2015; Powelson, 2011).

\section{PERFORMATIVITÉ AFFECTIVE}

II nous faut alors revenir à la question des affects. Selon Ashcraft, pour l'individu expérientiel ou affectif, les coûts de l'évaluation et du contrôle de la recherche semblent démesurément élevés. Voici comment elle dépeint certains affects produits:

La Règle de l'excellence est une force productive, disent-ils. Elle produit des choses, ou nous oblige à les faire. Les mots et les chiffres notamment. Elle produit ce savoureux hit, leur facteur d'impact tueur, ma marketabilité incomparable, notre ego gonflé, de longues queues pour avoir le poste, des cocottes-minutes qui débordent et des intérimaires pour prendre encore plus de tâches, plus d'étudiants, je veux dire des heures équivalent-TD (...) notre institution imitant la suante et haletante quête de la marque tout à fait distinctive et exactement identique (...) Ça aide à oublier ses sousproduits, comme l'anxiété, le stress, la détresse s'installant en dépression, les ulcères chroniques et autres maux de tête, et le jeu olympique virtuel du cynisme académique. La Règle applaudit ces tours de magie grâce auxquels nous atteignons son étalon, comme représenter la réalité depuis une bonne distance et avec un regard divin. Comme l'ironie nerveuse et un verre de plus. Comme faire comme si tout semblait sain. La Règle de l'Excellence prend son envol sur le moment. Parfois, nous la croyons sans hésitation, parfois nous nous débattons de son emprise, parfois nous la brandissons contre les autres. (Ashcraft, 2017, p. 49)

Le milieu universitaire vit visiblement un régime de performativité, où les apparences d'efficacité, d'habileté et de compétence sont plus importantes 
que la substance. L'extrait autobiographique suivant décrit l'expérience d'un des auteurs vécue dans une école de commerce très bien cotée :

«Première semaine de septembre, la rentrée allait débuter dans dix jours. J'avais été au loin pendant un mois, dans une ferme en Bourgogne où je m'étais délecté avec l'œuvre de Baudrillard, pour un chapitre de livre à écrire. Le plaisir et la concentration de s'immerger dans une œuvre, avec juste un écrivain, devaient maintenant prendre fin pour laisser place à l'enseignement et aux réunions des professeurs que je redoutais, où l'on devait constamment se méfier de la prochaine menace contre son espace intellectuel. Quelqu'un allait sûrement essayer de supprimer votre électif ou de vous forcer à enseigner des choses que vous croyez stupides, non pertinentes ou fastidieuses.

Guerre permanente, haine des collègues, exécration et peur étaient sur le point de recommencer. J'étais venu ce jour-là à Rotterdam car je savais que personne ne serait présent - c'étaient encore les vacances. Je pouvais aller chercher mon courrier (à l'époque il y avait encore cette sorte de chose) et me réhabituer sans avoir à réagir à quelqu'un. Et je saurais, sans que personne ne puisse me voir, si mon dernier article avait ou non été accepté.

J'ai marché jusqu'à mon bureau au rez-de-chaussée et découvert un autre nom sur la porte; et mes livres, mes documents et mon ordinateur n'étaient plus là. La gigantesque affiche du Holland Festival n'était plus sur le mur ; le bureau était dans une position différente; en fait, mon bureau en bois avait disparu, remplacé par un autre en métal. J'ai eu un moment de panique. Avaient-ils vraiment trouvé un moyen de me virer? Je savais qu'il y avait beaucoup de ressentiment contre moi, j'avais publié beaucoup plus qu'eux. N'était-ce pas une manière pas trop subtile d'annoncer ma fin à l'Université Erasmus? Je savais que j'avais des ennemis, quel universitaire n'en a pas?

La panique s'est progressivement effacée et je me suis rappelé d'une vague discussion d'avril dernier à propos de la relocation de tous les bureaux et des groupes de recherche dans l'immeuble. Une montée en ascenseur, un peu de recherche, et j'ai trouvé une porte avec mon nom. " (extrait autoethnographique)

Pareils doute de soi, hostilité et monde des conflits seront ce que le chercheur devra affronter tant que l'école de commerce sera plongée dans le bain des classements et du marketing. Plus de classements ne feront probablement qu'accroître la peur et la désignation de boucs émissaires. Willmott (2011) a psychologisé la question en parlant du « fétichisme des listes » et de ses effets en tant qu' "asphyxie auto-érotique ». Les affects ordinaires de l'autopromotion (self-branding) académique dans la société contemporaine 
dominée par le marketing sont profondément inquiétants, et à la limite autodestructeurs.

Bien sûr, certains s'épanouissent dans cette culture du jeu et de la prétention, mais ceux qui se préoccupent davantage de « citoyenneté, d'équité, d'inclusion sociale, d'investissement social et de construction nationale » en souffrent (Jessop, 2017:856). Dans l'école de commerce susmentionnée, des recherches ont été consacrées à des problèmes tels que l'acheminement des chariots élévateurs dans un entrepôt de brasserie ou la mise en œuvre des TIC dans le port de Rotterdam. Ces recherches visaient à allier performance commerciale et la production universitaire. Je ne peux pas (n'ai pas pu) m'identifier à ce régime de la répétition du succès où toute destruction serait créative. Les justifications de Schumpeter pour le chômage, la crise économique et les conflits sociaux ne m'ont jamais convaincu.

Mon expérience la plus saisissante et la plus humiliante à l'école de commerce advint lorsque j'enseignais le cours de gestion des ressources humaines en MBA. Les étudiants devaient lire Karen Legge (1995), un classique de GRH. Le cours précédent de comportement organisationnel reposait sur des cas avec des objectifs d'apprentissage fort modestes. Si les élèves comprenaient que l'organisation informelle était souvent plus puissante, que régulièrement la forme ne suit pas la fonction et que le pouvoir n'agit pas nécessairement de façon rationnelle, ils avaient compris le message. Le professeur était un excellent conteur et son QCM n'avait pas changé depuis des années; des rumeurs circulaient selon lesquelles les étudiants en avaient même des copies. Ainsi à rebours de la coutume, le cours de GRH était le plus sérieux. Je savais qu'en choisissant un texte de troisième année d'études universitaires britanniques, j'allais très loin. L'examen à livre ouvert au cours duquel les étudiants étaient invités à réfléchir sur leurs propres expériences, avait produit de nombreux textes fascinants. Mais cette année-là, les étudiants ont décidé de se rebeller. Ils étaient bien organisés et sans pitié. J'étais complètement à côté et sans pertinence en prétendant que le conflit entre employeurs et employés était une question importante. L'idée d'une possible crise économique était stupide. Répugnante était l'insistance à soutenir que les entreprises et la rhétorique du conseil en management étaient égoïstes, souvent malavisées. Inexcusable était de prétendre que la réduction des effectifs et la réorganisation reviendraient ou que la $\mathrm{GRH}$ était en soi un domaine contesté. J'étais incompétent, stupide, mal informé et c'était une honte, ils n'allaient pas faire l'examen. Le stratagème était clair, ils avaient décidé de ne pas faire le travail ou de ne pas lire le livre. Mais c'était le MBA et les étudiants des clients qui avaient payé. Par définition, j'avais donc tort et ils avaient raison. Le cours m'a été retiré et je n'ai pas eu le droit de l'enseigner à nouveau. J'ai été écarté, en larmes je dois l'admettre, par les étudiants triomphants, qui, je crois, s'en 
sont tirés en ne passant pas l'examen. L'année suivante, le cours de GRH a été donné par une consultante sans sensibilité académique ni critique, et les étudiants l'aimaient. (extrait autoethnographique)

Quels sont les effets du néolibéralisme pour un universitaire critique ? Pour les croyants, la logique néolibérale principal-agent est tout à fait acceptable. Les relations sont contractuelles, les deux parties s'efforçant de maximiser leurs propres avantages. Le professeur de comportement organisationnel était un maître en la matière - il a gardé les étudiants heureux et amusés, et ils ont appris quelque chose. II était totalement indifférent aux questions de justice, d'équité ou d'inclusivité citoyenne. Tant qu'eux et lui étaient heureux de leur situation, tout allait bien. II aimait bien manger et boire de bons vins.

Dans la culture de l'école de commerce, montrer de l'émotion était un signe de faiblesse. Les cultes de la performance et du succès étaient la norme. Le turnto-affect dans la recherche (Clough, 2007 ; Stewart, 1996) était une donnée aberrante. En effet, la question «Quels sont les affects dominants dans ce monde universitaire ? " relève dans un vocabulaire deleuzien du " devenir mineur ». Et pourtant, il y a des universitaires dans ces mêmes écoles dont les écrits ne correspondent pas au modèle performatif dominant, à l'image de Beyes et Steyaert :

\begin{abstract}
Puis-je décrire en mots ou en images comment j'entre chaque jour par la porte de mon bureau d'où j'écris maintenant cette phrase ? Puis-je rejouer cette scène d'entrée au ralenti et rendre visible tous les affects, les matériaux, les mouvements qui se nouent à ce moment ? Est-ce que je peux relier dans cette description le rythme régulier de mes doigts sur le clavier, les frappes à la porte deux secondes plus tôt par deux collègues me proposant de déjeuner avec eux, les échos de l'appel enthousiaste du co-auteur de ce texte avec qui nous avons envisagé la possibilité de modifier le style et d'interrompre notre rédaction pour le prochain match de football télévisé, les camions qui passent sous ma fenêtre pour livrer pierres, débris et matériaux de construction sur le campus pour la rénovation complète du bâtiment de l'université, les ombres projetées sur les murs blancs et tapis gris créés par les rayons de lumières envoyés par le soleil, le sens de l'horizon que me donne un regard par l'autre fenêtre d'où j'aperçois le lac ? (...) Peut-on faire revivre notre prise dans des devenirs spatiaux plutôt que de la décrire avec nos procédés normalisées de création de connaissances qui bâtissent de la distance ? (Beyes \& Steyaert, 2011, p. 46)
\end{abstract}

Cette citation explore des possibilités habituellement inexprimées. Elle tente d'entrer dans «les intensités, les capacités et forces; rythmes, cycles, rencontres, événements, mouvements et flux ; instincts, affects, atmosphères et auras ; relations, nœuds et agencements » (idem, p. 46). La différence entre 
l'arrivée de Beyes et de Steyaert dans leur bureau et celui du bureau disparu est qu'il y avait beaucoup plus de défamiliarisation dans le second. Quelle rupture pourrait être plus grande que ne plus avoir de bureau dans ce que vous considérez comme votre lieu de travail ? Beyes et Steyaert cherchent à devenir mineurs ou hyperdiachronisés : à aller toujours plus loin en essayant d'être novateurs, intéressants et expérimentaux. Ils s'efforcent d'être « l'autre voie empruntée » et de se faire les champions de "l'affect fugace ». Leur texte est certes attractif mais il évite de se confronter au majeur, au courant dominant institué, la performance des entreprises et le capitalisme académique. Malheureusement, Beyes et Steyaert restent ainsi à un méta-niveau. Ils pointent une possibilité épistémologique, mais que peut-on faire avec cette possibilité ? Dans une école de commerce, la réponse pourrait bien être : « pas grand-chose ». II y a été admis qu'il fallait maximiser la liberté des acteurs économiques, la connaissance au service du bien public a été délégitimée. Dans l'épistémè néolibéral dominant, les idées socialement acceptables sont assez limitées et tout appel à agir tabou.

Je l'ai vécu comme un environnement d'impermanence dirigé par la peur, lourd de constantes menaces et d'une concurrence effrénée. Si l'on publiait un article dans une revue critique et/ou qualitative, les collègues tentaient immédiatement de retirer ce journal de la liste officielle. Le temps de recherche était constamment menacé, il fallait se battre pour conserver ses modules avancés. Lorsque je suis parti ailleurs pour un poste avec chaire, un professeur sénior m'a informé, avec un sourire narquois, qu'il savait que le département vers lequel je me dirigeais devait être dissous par le Ministère de l'Éducation, ce qui n'était pas vrai. Il y avait une rumeur constante de réorganisations, qui n'est jamais venue. Certaines personnes pouvaient faire leurs heures de travail de façon extravagante et d'autres étaient surmenées. II n'y avait pas de transparence et une intimidation constante. Le leadership était autoritaire. Les professeurs avaient d'énormes privilèges (semi-secrets) et des budgets personnels, tandis que les maîtres de conférences étaient exclus de tous les avantages. J'y repense avec frustration et colère. Pour une part, cela vient que je n'étais pas adapté, vous deviez soutenir la politique néolibérale. Après tout, le rapport sur la compétitivité de la région pour le Forum Économique Mondial avait été finalisé ici. (extrait autoethnographique)

Une carrière minoritaire n'est pas facile, cela demande le goût de l'expérimentation, de jouer avec les textes et de rester non conventionnel. Cela vous expose très probablement au ridicule, à l'insécurité d'emploi et à 
l'agressivité. Et même si des amis universitaires parviennent ensemble à produire des textes très personnels, individuels et uniques, seront-ils plus proches d'un «Que faire ensuite ? ». Bien sûr, la critique, pour être puissante et appréciée, n'a pas à offrir une alternative. Mais en appeler à un «nous » d'une implication attentionnée, d'une participation engagée et d'une communauté partagée peut être juste un moyen de réaffirmer I'hyperdiachronisation. Ensemble nous ressentons la solidarité, la chaleur, l'amitié et la collégialité, alors que le néolibéralisme nous menace. Une éthique de la recherche participative dans le cadre d'une " économie de contribution » tente bien de contrecarrer la " mégamachine » de l'entreprise, mais est-ce plus qu'une simple voix qui crie dans le désert? Paradoxalement, nous semblons vouloir que l'affect anti-performatif soit performatif ! Sinon, ne ferait-on pas mieux d'accepter l'hégémonie néolibérale et le régime du capitalisme académique comme nôtres ?

\section{POUR CONCLURE : QUELLE EST LA PROCHAINE ÉTAPE ?}

Dans cet article, nous avons présenté la littérature critique sur les classements des revues et des départements, en mettant l'accent sur les coûts de la « quête de l'excellence ». Nous croyons que la performativité qui transit les écoles de commerce est en contradiction avec une bonne recherche en sciences sociales. Les classements font partie du marketing de l'enseignement supérieur (managérial). La recherche comme outil de marketing sert à différencier les écoles de commerce, le $4 \times 4^{*}$ académique faisant partie de la stratégie.

Certains textes académiques s'ouvrent aux sentiments et à la prise de conscience. Ils s'opposent à la domination du cliché, de la conformité et du managérialisme. Ils sont la preuve qu'il est possible de réaliser quelque chose de minoritaire, différent de ce qui se publie le plus facilement. Dans les textes qui montrent les affects académiques, y a-t-il quelque chose de plus que colère, frustration et douleur? Oui, nous pouvons pour un moment nous ouvrir aux affects, mais «l'affect ordinaire » (Stewart, 2007) de l'école de commerce n'est-il pas répressif, performatif et hostile aux études sociales ? La vie à l'école de commerce est-elle traumatisante, car la valeur 
du travail et des œuvres est rejetée?

Alors malgré les critiques justifiées de Stiegler sur l'hyperdiachronisation, existe-t-il une meilleure alternative que celle de former sa propre petite communauté de travail coopératif et de la protéger de tout le reste $?^{3}$ Les classements et les notations sont l'épiphénomène, le capitalisme académique au sein du régime du néolibéralisme est plus fondamental. Mais l'économie de la connaissance est le principal acte. Si nous supposons que la seule façon pour l'Occident postindustriel de faire face à la désindustrialisation est d'être économiquement plus enraciné dans le savoir, innovateur et créatif que les autres, alors la redéfinition de l'université comme terreau pour un renouveau de l'économie suit logiquement.

Dans cette perspective, les affects ne sont pas seulement des effets secondaires mais une question centrale. Les universitaires craintifs, effrayés, peu sûrs d'eux-mêmes ne peuvent pas innover et ne le feront pas. L'innovation n'est pas efficacement guidée par la peur. Le paradoxe majeur est que les grandes entreprises sont hyper-hiérarchiques et que la voix du patron fait loi. Mais ce n'est pas cette logique qu'il vous faut pour être créatif. L'affect ne devrait pas être un sous-thème marginal mais participer de l'acte principal. Aujourd'hui, la recherche interrogeant les affects est marginale, juste tolérée. Mais nous affirmons que la corrélation entre les affects, le renouveau et l'innovation est cruciale. Aujourd'hui, trop de recherches dirigées par les affects soulignent l'insatisfaction et la terreur du subalterne; qui n'a pas voix. Le contraire, la célébration de l'affect en tant que capacité partagée est une nécessité urgente. Certes nous admettons que nous n'avons pas produit un tel texte positif par cet article. En effet, le discours dominant est répressif, craintif, compétitif et vindicatif. Un programme de soutien, de solidarité intellectuelle et de coopération reste manquant. Les analyses de Stiegler, aussi puissantes soient-elles, éclairent beaucoup plus ce qui est destructeur et mauvais que des alternatives. Pour l'instant, tout ce que nous pouvons faire, c'est demander à cette revue d'annoncer un autre numéro spécial sur le travail coopératif, la réussite de l'individuation

${ }^{3} \mathrm{Cf}$. le programme « le sens dans les organisations », présenté dans Letiche \& Lightfoot (2014) et Letiche, Lightfoot \& Moriceau (2016). 
académique et le rôle dans cela des affects de l'entraide, de l'innovation et de la créativité.

\section{RÉFÉRENCES}

Anonymous (2015) "Why I had to quit the research excellence research panel" Times Higher Education November 19.

Arendt, H (1998) The Human Condition Chicago: University of Chicago Press.

Ashcraft, K (2017) 'Submission' to the rule of excellence: Ordinary affect and precarious resistance in the labor of organization and management studies" Organization 24 (1) 36-58.

Beyes, T, Steyaert, C (2011) "Spacing Organization" Organization 19 (1) 45-61.

Boesenberg, E (2015) "Survival in the New Corporatized Academy" Workplace: A Journal for Academic Labor, No 25.

Butler, N \& Spoelstra, S (2014) "The Regime of Excellence and the Erosion in Critical Management Studies" British Journal of Management 25 (3) 538550.

Cantwell, B \& I Kauppinen (eds) (2014) Academic Capitalism in the Age of Globalization Baltimore: John Hopkins Press

Carter, C (2008) "A Curiously British Story" International Studies of Management and Organization 38 (1) 14-30.

Cederstrom, C \& Hoedemaekers, C (2012) "On dead dogs and unwritten jokes: Life in the university today" Scandinavian Journal of Management 28 (3) 229-233.

Clough, P \& J Halley (eds) The Affective Turn Durham NC: Duke University Press.

Cooper, C \& Otley, D (1998) "The 1996 Research Assessment Exercise for business and management' British Journal of Management 9 (2) 73-89.

Dillet, B (2017) "Proletariatization, Deproletariatization, and the Rise of the Amateur" boundary2 vol 44 no 1 pp 79-105.

Duménil, G \& D Lévy (2004) Capital Resurgent London: Harvard University Press.

- $\quad$ (2011) The Crisis of Neo-Liberalism London: Harvard University Press.

Fournier, V \& Grey, C (2000) "At the critical moment: conditions and prospects for CMS" Human Relations 53 (1) 7-32.

Furlong, J (2011) "Universities and the Discipline of Education: understanding the impact of the United Kingdom's Research Assessment Exercise" Power \& Education 3 (1) 18-30.

Gonzales, L, E Martinez \& C Ordu (2014) "Exploring Faculty Experiences in a striving university through the lens of academic capitalism" Studies in Higher Education vol 39, no. 7, pp 1097-1115.

Graeber, D (2011) Debt: The first 5,000 years Londoin: Melville House Hall, C (2011) "Publish or Perish: Bibliometric Analysis" Tourism Management 32 16-27.

Harvey, D (2005) A Brief History of Neoliberlaism Oxford: Oxford University Press.

Hughes, S (2011) Genetech: the beginnings of biotech Chicago: University of Chicago Press.

James, W (1920) The Letters of William James Boston: Atlantic Monthly Press. Jessop, B (2017) "Varieties of academic capitalism and entrepreneurial 
universities" The Journal of High Education vol 73 no 6 pp 853-870.

Letiche, H, Lightfoot, G (2014) The Relevant PhD Rotterdam: Sense Publishing. Letiche, H, Lightfoot, G, Moriceau, J-L (2016) Demo(s) Rotterdam: Sense Publishing.

Letiche, H \& J-L Moriceau (2017) "Simondon: Investigating the pre-indivdual" Culture \& Organization vol 23 no 1 pp 1-13.

Lilley, S (2017) Oral communication.

Macdonald, S, Kam, J (2007) Ring a Ring 'o Roses: Quality Journals and Gamemanship in Management Studies" Journal of Management Studies 44 (4) 640-655.

Marcus, G (ed) (1995) Technoscientific Imaginaries Chicago: University of Chicago Press.

Mingers, J, \& Willmott, H (2013) "Taylorizing business school research, on the 'one best way' performative effects of journal ranking lists" Human Relations 66 (x) 1051-73.

Morris, H, Harvey, C, Kelly, A (2009) "Journal rankings and the ABS quality guide" Management Decision 47 (9) 1441-51.

Naidoo, R (2016) "The Competition Fetish in Higher Education" British Journal of the Sociology of Education vol 37, no 1, pp 1-10.

Powelson, M (2011) "The Proletarianization of the Academy" Workplace: $A$ Journal for Academic Labor no 18.

RAE (2006) Panel criteria and working methods: 138 Business and Management Studies, accessed 30 January 2017 available at: http://www.rae.ac.uk/pubs/2006/01/docs/i36.pdf,

RAE (2008) Subject Overview Reports: 136 Business and Management Studies, accessed 30 January 2017 available at: http://www.rae.ac.uk/pubs/2009/ov.

Rhoades, G (2014) " Extending academic capitalism by foregrounding academic labor" in Cantwell, B \& I Kauppinen (eds) Academic Capitalism in the Age of Globalization pp 113-134, Baltimore: John Hopkins Press

Sayer, D (2015) Rank Hypocrisies London: Sage.

Scott, D (2014) Gilbert Simondon's Psychic \& Collective Individuation Edinburgh: Edinburgh University Press.

Slaughter, S \& L Leslie (1997) Academic Capitalism: Politics, Policies and the Entrepreneurial University Baltimore: John Hopkins Press

- (2001) "Expanding and Elaborating the Concept of Academic Capitalism Organization vol 8 no 2 pp 154-161.

Slaughter, S \& G Rhoades (2004) Academic Capitalism and the New Economy Baltimore: John Hopkins Press.

Stewart, K (1996) A Space on the Side of the Road New Haven: Princeton University Press.

-- (2007) Ordinary Affects Durham NC: Duke University Press

Stiegler, B (1998) Technics \& Time no 1 Palo Alto: Stanford University Press.

- $\quad$ (2008) Technics \& Time no 2 Palo Alto: Stanford University Press

- $\quad$ (2010a) Technics \& Time no 3 Palo Alto: Stanford University Press

- $\quad$ (2010b) Taking Care of Youth and the Generations Stanford: Stanford University Press.

- $\quad$ (2013) Pharmacologie du Front National Paris: Flammarion. 
- $\quad$ (2016) Dans la disruption Paris: Éditions Les Liens Qui Libèrent.

Strathern, M (1997) "'Improving ratings' audit in the British University System" European Review 5 305-21.

Sum, N-L \& B Jessop (2013) "Competitiveness, the Knowledge-BasedEconomy \& Higher Education" Journal of Knowledge Economy vol 4 no 1 pp 24-44.

Tadajewski, M (2016) "Academic labour, journal ranking lists and the politics of knowledge production in marketing" Journal of Marketing Management $32(1-2) 1-18$.

Tourish, D, \& Willmott, H (2014) "In Defiance of Folly: Journal Rankings, mindless measures and the ABS Guide" Critical Perspectives in Accounting 26 (1).

Walker, J (2009) "Time as the fourth dimension in the globalization of higher education" The Journal of Higher Education vol 80 no 5 pp 483-509.

- $\quad$ (2014) " "Exploring the academic capitalist time regime" in Cantwell, B \& I Kauppinen (eds) Academic Capitalism in the Age of Globalization pp 55-73, Baltimore: John Hopkins Press

Ward, S (2012) Neoliberalism and the global restructuring of knowledge and education New York: Routledge.

Willmott, H (2011) "Journal list fetishism and the perversion of scholarship: reactivity and the ABS list" Organization 18 (4) 429-42.

- (2014) "In defiance of folly: Journal rankings, mindless measures, and the ABS guide" Critical Perspectives on Accounting 26 (1) 37-46. 